Revue internationale P.M.E.

Économie et gestion de la petite et moyenne entreprise

Revure

internationale

PME

\title{
Les effets du financement public sur les entrepreneurs et leurs entreprises : le cas des PME du Québec
}

\section{Jocelyn J-Y. Desroches et Vijay M. Jog}

Volume 2, numéro 1, 1989

URI : https://id.erudit.org/iderudit/1007905ar

DOI : https://doi.org/10.7202/1007905ar

Aller au sommaire du numéro

Éditeur(s)

Presses de l’Université du Québec

ISSN

0776-5436 (imprimé)

1918-9699 (numérique)

Découvrir la revue

Citer cet article

Desroches, J. J. \& Jog, V. M. (1989). Les effets du financement public sur les entrepreneurs et leurs entreprises : le cas des PME du Québec. Revue internationale P.M.E., 2(1), 19-37. https://doi.org/10.7202/1007905ar

\section{Résumé de l'article}

Un sondage mené auprès de nombreux PDG de PME devenues publiques grâce au programme québécois du RÉA, sondage s'étalant de 1984 à 1987, est à l'origine de ce document de recherche qui vise essentiellement à évaluer les effets de la décision de recourir au financement public sur le statut de l'entreprise, sa gestion interne et son PDG en tant qu'individu. Les résultats de cette enquête démontrent que la majorité des PDG qui ont répondu sont très satisfaits de leur décision. Selon eux, l'influence la plus grande et la plus importante porte, surtout, sur le statut de l'entreprise qui devient alors crédible aux yeux du monde extérieur. D’autres effets importants et positifs sont aussi ressentis surla structure et sur les communications en général. Contrairement à la croyance populaire, les PDG de nos entreprises devenues publiques ne perdent pas le contrôle de leur entreprise; ils deviennent beaucoup plus intéressés à adapter leur structure aux nouveaux besoins et à rationaliser leur processus de prise de décision. Que les dirigeants n’apprécient guère l'évaluation de la valeur en bourse des actions de leur entreprise ne change pas la conclusion générale de cette recherche : devenir une société ouverte implique des changements significatifs quant au statut de l'entreprise, à sa structure administrative et chez son dirigeant. Presque tous ces changements sont positifs et favorisent une telle orientation.
Ce document est protégé par la loi sur le droit d'auteur. L'utilisation des services d'Érudit (y compris la reproduction) est assujettie à sa politique d'utilisation que vous pouvez consulter en ligne.

https://apropos.erudit.org/fr/usagers/politique-dutilisation/ 


\title{
Les effets du financement public sur les entrepreneurs et leurs entreprises : le cas des PME du Québec
}

\author{
Jocelyn J-Y. DESROCHES*, \\ Université du Québec à Montréal \\ Vijay M. JOG**, \\ Université Carleton
}

\begin{abstract}
RESUME
Un sondage mené auprès de nombreux PDG de PME devenues publiques grâce au programme québécois du RÉA, sondage s'étalant de 1984 à 1987, est à l'origine de ce document de recherche qui vise essentiellement à évaluer les effets de la décision de recourir au financement public sur le statut de l'entreprise, sa gestion inteme et son PDG en tant qu'individu. Les résultats de cette enquête démontrent que la majorité des PDG qui ont répondu sont très satisfaits de leur décision. Selon eux, l'influence la plus grande et la plus importante porte, surtout, sur le statut de l'entreprise qui devient alors crédible aux yeux du monde extérieur. D'autres effets importants et positifs sont aussi ressentis surla structure et sur les communications
\end{abstract}

- Le professeur Desroches est titulaire d'un doctorat (Ph. D.) de l'Université de Toronto. Ses domaines de recherche incluent la gestion des PME, les sources alternatives de financement des PME en croissance et la relation entre les gouvernements et le secteur privé. Il est responsable de la section management de son département. Adresse: Département des Sciences administratives, Université du Québec à Montréal, Case postale 8888, succursale A, Montréal, Québec, H3C 3P8.

** Le professeur Jog possède un Ph. D. en Finance de l'Université Mc Gill. Ses domaines de recherche et de consultation incluent la finance corporative, les programmes gouvemementaux, l'entrepreneuriat, les sociétés de capital de risque et l'évaluation d'entreprises. Adresse: Département de finance, Université Carleton, Ottawa, Canada, K1S 5B6.

Les auteurs désirent remercier l'Institut de recherches politiques du Canada, la Bourse de Montréal et la Bourse de Toronto pour leur commandite qui leur a permis d' effectuer un ensemble de recherches sur les petites et moyennes entreprises et leur financement public au Canada. Les conclusions répertoriées dans ce document sont tirées en partie d'un important ouvrage qui sera publié au début de 1990. Première version reçue en juin 1989. 
Les effets du financement public sur les entrepreneurs et leurs entreprises

en général. Contrairement à la croyance populaire, les PDG de nos entreprises devenues publiques ne perdent pas le contróle de leur entreprise; ils deviennent beaucoup plus intéressés à adapter lour structure aux nouveaux besoins et à rationaliser leur processus de prise de décision. Que les dirigeants n'apprécient guère l'évaluation de la valeur en bourse des actions de leur entreprise ne change pas la conclusion générale de cette recherche : devenir une société ouverte implique des changements significatifs quant au statut de l'entreprise, à sa structure administrative et chez son dirigeant. Presque tous ces changements sont positifs et favorisent une telle orientation.

\begin{abstract}
The study documents results of a survey conducted with the CEOs of small and medium-sized businesses which went public between 1984 and 1987 using the QSSP (Quebec Stock Savings) Program. The emphasis of the research reported here was to evaluate the impact of the going public decision on three specific dimensions : the status of the business, the management of the business, and on the CEO as an individual. The results of this research indicate that an ovenwhelming majority of the CEOs who responded to the survey consider their decision to go public in a very favorable light. The largest and most important impact of going public, according to them, is on the status of the business itself-itimproves significantly as seen by outsiders; it is followed by the impact on the management structure of the business, and communications in general. Contrary to common beliefs, CEOs do not convey a significant loss of control and actually welcome the more structured and decision-making resulting from going public. Although they do not like the valuation of their shares by the market place, the overall conclusion of this research remains unchanged : going public does imply significant changes to the status of the firm, management structure, and the entrepreneur, but almost all these changes are positive and welcomed by those who took their firms public.
\end{abstract}

\title{
RESUMEN
}

El estudio fue realizado con un centenar de dirigentes de empresas medianas y pequeñas (PyME) que se volvieron publicas en acuerdo con el programa REA entre 1984 y 1987. Este documento tiene como objetivo la evaluacion del impacto de la financiacion publica sobre tres niveles, o sea el statuto de la empresa, su gestion interna y su dirigente como individuo. Los resultados de este estudio demuestran que la mayoria de los dirigentes que han contestado a nuesta encuesta estan muy satisfechos de haber tomado la decision de acudir al financiamiento publico. Segun ellos, el impacto mas grande e importante es el que se hace sentir sobre ol estatuto de la empresa que se transforma en una entidad mucho mas importante a los ojos del mundo exterior. Otros efectos importantes y positivos se recienten al nivel de la estructura de la empresa y de las comunicaciones en general.

Contrariamente a la opinion general los dirigentes de las PyME que se transformaron en empresas publicas dicen no haber perdido el control de su empresa; haber optado por una posicion mas flexible para ajustar las estructuras a las nuevas necesidades y para racionalisar el proceso de toma de decision en la empresa Aunque los dirigentes no aprecian la valuacion de las acciones de su empresa en el mercado bursatil, la conclusion de esta investigacion es : transformarse en una empresa publicaimplica cambios significativos sobre el estatuto de la empresa, su structura administrativa y su dirigente. Casi todo los cambios son positivos favoreciendo por consiguiente una tal orientacion. 


\section{Introduction}

Deux phénomènes ontmarqué l'évolution industrielle des sociétés modernes au cours des quinze dernières années. Ce sont, d'une part, la reconnaissance du secteur privé comme véritable moteur économique et, d'autre part, le poids de plus en plus prépondérant des petites et moyennes entreprises (PME) à l'intérieur de ce même secteur. Grâce à leur propension à l'innovation, à leur flexibilité technologique età leur capacité d'adaptation aux besoins ambiants, ces entreprises, qui généralement comptent moins de 200 employés, sont devenues les plus grandes créatrices d'emplois nets «au Québec, au Canada, en Amérique et ailleurs dans le monde» (Tardif, 1985: 1; Smith, 1984: 4; Bulloch, 1985).

Conscient de l'importance de la vitalité et de la croissance des PME pour son économie, le gouvernement québécois, à l'instar des autres provinces canadiennes, créa plusieurs programmes visant l'amélioration de leur gestion, l'expansion de leurs équipements et de leurs immobilisations, ainsi que la rentabilité de leur situation financière. Parmi ces programmes, le plus innovateur et le plus «entrepreneurial», selon l'avis des experts, a été mis sur pied par le Québec. Le Régime d'épargneactions (RÉA), introduit en 1979, permet aux personnes qui investissent dans les entreprises québécoises accréditées par le RÉA, donc cotées à la Bourse, de bénéficier d'une déduction fiscale avantageuse reliée à cet investissement.

Le programme s'est donné deux objectifs principaux : foumir le capital propre (capital equity) dont les PME dynamiques du Québec ont un grand besoin pour se développer et encourager les petits épargnants à s'intéresser à un nouveau véhicule d'investissement de leurs économies, celui des PME québécoises.

Depuis maintenant dix ans, l'instauration du RÉA a permis à 350 entreprises du Québec d'augmenter leur avoir propre de quelque dix milliards de dollars. De ce montant, environ deux milliards de dollars ont bénéficié directement à $178 \mathrm{PME}^{1}$ enregistrées à la Bourse de Montréal entre 1984 et 1987. Le succès du programme RÉA fut tel que les médias le considérèrent rapidement comme la source d'une révolution entrepreneuriale au Québec et au Canada.

Malgré plusieurs études et rapports effectués sur la performance du marché boursier grâce à la venue de nouvelles entreprises bénéficiant du RÉA, nous trouvons peu de recherches sur certains volets, tels que :

a) les critères qui ont influencé les entrepreneurs dans leur décision d'opter pour le financement public;

b) les motifs qui ont incité ces entrepreneurs à procéder au changement du statut légal de leur entreprise, $c$ 'est-à-dire de transformer leur société fermée en société

1. Il s'agit ici d'entreprises appelées «Sociétés en voie de développement» qui ont un capital net égal ou inférieur à 25 millions de dollars avant de devenir une entreprise ouverte. 
ouverte $^{2}$, ce qui implique une surveillance rigoureuse de la part du gouvernement et un contrôle accru du conseil d'administration;

c) et, principalement, l'influence de cette décision sur les entrepreneurs euxmêmes en tant qu'individus; sur leur entreprise pour ce qui concerne la structure, les relations de travail, le processus décisionnel, les communications, etc.

Cette recherche se concentre essentiellement sur ce troisième aspect, plus précisément sur les effets de la décision par ces entrepreneurs de recourir au financement public. La recherche repose sur un sondage effectué auprès de ces entrepreneurs.

\section{Le contexte}

Avant de présenter les différents aspects de l'entreprise et de l'entrepreneur, lesquels seront traités selon une approche qualitative, il parait opportun de situer cette recherche dans son contexte et de faire part de la disponibilité de l'information sur le sujet. Il est nécessaire de mentionner qu'il ne semble exister aucune recherche établissant clairement une preuve directe de l'influence des différents aspects qui font l'objet de la présente étude. Aussi, nous avons peu d'indications nous permettant d'obtenir un consensus sur la compréhension des facteurs déterminants qui facilitent l'accès au financement public par les PME et l'influence de ce financement public sur les entreprises et leurs entrepreneurs.

Un examen de la documentation financière, des écrits sur l'entrepreneuriat et des études et commentaires sur les PME ont été mis à contribution comme fondement de cette recherche afin de juger de la pertinence de trois ensembles de variables et ainsi permettre de dégager les principaux facteurs en jeu. Comme nous le verrons, cés facteurs sont reliés à l'environnement externe, aux caractéristiques de l'entreprise et à celles de l'entrepreneur.

\subsection{L'environnement financler des PME}

La Commission québécoise sur la capitalisation des entreprises (1984) et le Conseil économique du Canada (1982) ont démontré que les principaux obstacles au financement public des PME relèvent à la fois du système fiscal, du fonctionnement des institutions financières (banques, fiducies, caisses populaires, groupes de crédit), de l'industrie des valeurs mobilières elle-même et, finalement, de la culture et des

2. Les termes «sociétés ouvertes et sociétés publiques» ont dans ce textelamêmesignification : ce sont des entreprises qui ont modifié leur statut légal afin d'avoir accès au financement public. Les termes «sociétés fermées ou privées* regroupent celles qui ne peuvent pas ou ne veulent pas recourir au financement public. 
traditions de la communauté des gens d'affaires québécois où il n'existe que quelques modèles qui ont émergé depuis l'établissement du programme. Par contre, les politiques fiscales des différentes autorités gouvernementales facilitent de plus en plus l'achat d'actions des PME qui décident de faire appel aux capitaux publics (Conseil économique du Canada, 1982; Commission québécoise sur la capitalisation, 1984; Guertin, 1984; Robert, 1983). Dès quel'entreprise devient une sociêté ouverte, des incidences sont donc à prévoir sur l'environnement financier de l'entreprise et son accessibilité au financement externe.

Deux thèmes semblent avoir retenu l'attention des analystes financiers et des chercheurs intéressés par le phénomène RÉA, à savoir la performance financière des entreprises RÉA cotées à la Bourse (Dubé, 1988; Sécor, 1986, 1988; Riverin 1988a; Chiasson, 1986; Henri, 1987; The Globe and Mail, 1984; The Financial Times of Canada, 1985) et la participation de la population québécoise à ce programme (Martin, 1987; Riverin, 1988b). Bien qu'ils reconnaissent que les entreprises devenues sociétés ouvertes soient obligées de faire face à un environnement externe différent, ces auteurs ne fournissent aucune preuve directe quant à l'étendue de ce changement, et plus spécifiquement quant à la perception des entrepreneurs qui ont opté pour un tel changement.

\subsection{Les caractéristiques de l'entreprise}

Plusieurs facteurs ont joué un rôle considérable dans la décision de devenir une société publique; parmi les plus importants, et reliésà la firme elle-même, on note les suivants : le stade de développement de l'entreprise (Steinmetz, 1974; Kostuch, 1974; Commission québécoise sur la capitalisation des entreprises, 1984), la taille de l'entreprise (Liao, 1975, Brigham and Smith, 1975), sa situation concurrentielle sur le marché (O'Neill, 1983), les coûts d'obtention du capital (Kostuch, 1976; Arnold, 1985) et le financement du capital propre (Thornton, 1981; Wynantet al., 1982; Dunn and Bradstreet, 1986).

L'intérêt principal de la présente étude est d'expliquer les raisons de la décision des entrepreneurs de convertir leur entreprise en société ouverte plutôt que de s'étendre sur les répercussions que connaît l'entreprise après une telle transformation. C'est donc l'ultime question qui sera analysée dans cette recherche.

\subsection{Les caractéristiques du propriétaire}

Au-delà des strictes considérations financières, l'idéologie del'entrepreneur (Toulouse, 1979; Gasse, 1983), ses valeurs et ses attitudes à l'égard de sa firme (Bamberger, 1986), son désir d'autonomie et de contrôle de sa firme (de Vries, 1977; Gasse, 1982; O'Neill, 1983), sa scolarité et sa formation en management (Gasse, 1985) sont parmi les variables personnelles souvent reliées à la décision de recourir ou non au financement public. 
24

Les effets du financement public sur les entrepreneurs et leurs entreprises

Toutefois, les études de Gasse (1979, 1984a, 1984b) sont probablement les plus pertinentes jusqu'à aujourd' hui en ce qui concerne les attitudes des entrepreneurs des PME québécoises face à l'option de transformer leur société fermée en société ouverte. On y montre, entre autres, que l'idée du financement public est récente chez nous et qu'elle est surtout le phénomène de firmes plus jeunes. Une question intéressante attire particulièrement notre attention, à savoir quelle est l'influence du statut d'une entreprise devenue société ouverte sur les dimensions individuelle et personnelle de l'entrepreneur. La mise à jour de cette influence pourrait aider les futurs entrepreneurs à évaluer la décision de devenir une société ouverte de façon plus officielle.

Quoiqu'aucun de cesécrits ne nous foumisse un modèleétoffé d'analyse des effets du financement public surl'entreprise, ils nous permettent cependant d'identifier trois pôles principaux influencés par ce changement de statut de l'entreprise, à savoir le statut de l'entreprise (pôle externe), la gestion de l'entreprise (pôle interne) et l'entrepreneur (pôle personnel). Le diagramme 1 fait état de ce cadre conceptuel abrégé.

Diagramme 1

Les différents póles du financement public

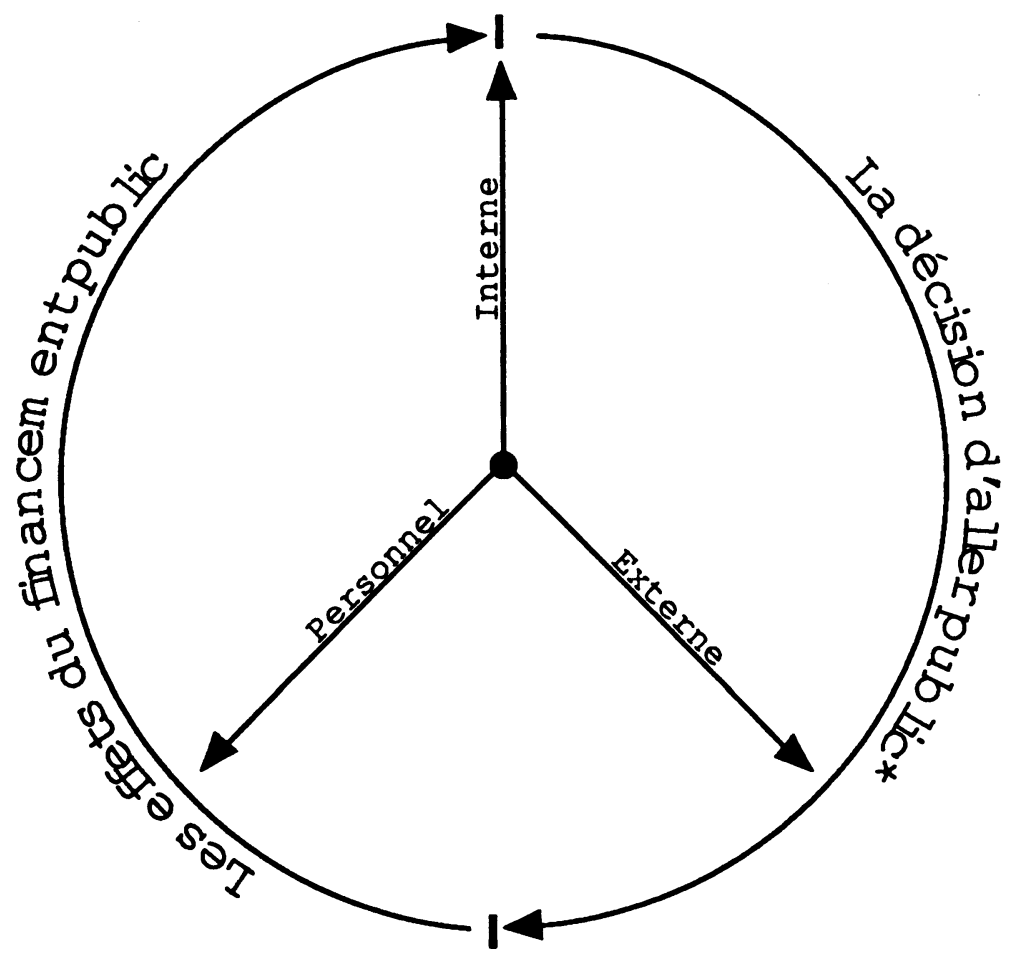

" La décision d’aller public signifie la décision de devenir une société ouverte. 


\section{Les avenues de la recherche et sa structure}

Parce qu'on suppose que les changements observables possibles sont de trois ordres : ceux reliés à la perception qu'ont les gens à l'extérieur de l'entreprise (le pôle externe); ceux reliés à la gestion de l'entreprise elle-même (le pôle interne) et ceux reliés au PDG lui-même en tant qu'individu (le pôle personnel) lesquels sont présentés au diagramme 1 (p. 24), les questions de recherche qui nous intéressent sont les suivantes :

1. À quel ordre appartiennent les changements les plus importants que suscite l'arrivée de fonds publics dans l'entreprise ? Sont-ils d'abord ressentis sur le plan interne, externe ou personnel ? (tableau 1)

2. Quels sont les types et les éléments de changements les plus marqués sur le plan interne, externe et personnel ? (tableaux 2 et 3, p. 27 et 29).

3. Est-ce que les changements les plus marqués sont perçus comme positifs ou négatifs pour le développement futur de la firme ? (tableau 4, p. 33).

Une typologie simple a été créée pour évaluer, d'une part, le niveau de changement et, d'autre part, le degré d'importance de six groupes de variables reliées à l'un ou à l'ensemble des différents pôles.

Tab. 1. Niveaux de changements perçus par le PDG public : une typologle

\begin{tabular}{|l|c|c|c|}
\hline \multirow{2}{*}{ Types de changements } & \multicolumn{3}{|c|}{ Trois póles } \\
\cline { 2 - 4 } & externe & interne & individu \\
\hline 1. Statut de l'entreprise & $\mathrm{X}$ & & \\
2. Restructuration de & & $\mathrm{X}$ & \\
l'entreprise & & $\mathrm{X}$ & \\
3. Communications en & & $\mathrm{X}$ & \\
4. général & & $\mathrm{X}$ & \\
5. Recessus de décision & & & $\mathrm{X}$ \\
6. PDG lui-méme en tant & & & \\
\hline
\end{tabular}

Comme le montre le tableau 1, ces trois pôles sont représentés par six groupes de variables. Concrètement, le pôle externe est constitué d'un seul groupe de variables touchant le statut externe de l'entreprise. Le pôle interne est, pour sa part, constitué de quatre groupes de variables en correspondance les unes avec les autres, mais on a voulu les séparer sur une base intuitive et par souci de clarté. Les types d'influence à la gestion interne de l'entreprise sont : la structure de l'entreprise, les communications en général, le processus de prise de décision et les relations de travail. Enfin, le pôle personnel est constitué d'un seul groupe de variables touchant le PDG lui-même en tant qu'individu. 
L'influence opérationnelle du financement public est par la suite mesurée par l'incidence perçue par le PDG de la présence ou l'absence d'un certain nombre d'éléments ou facteurs reliés à chacun des six groupes présentés ci-dessus. Les éléments du tableau 1 ont été choisis parce qu'ils semblaient importants et pertinents pour les experts consultés, pour un échantillon de PDG consultés au cours de la construction de notre questionnaire et lors du test préliminaire.

Comme l'objectif principal de cette recherche ${ }^{3}$ est l'étude de ces trois pôles dans le contexte de la prise de décision de l'entrepreneur de transformer son entreprise en société ouverte, nous avons concentré notre analyse sur ce groupe de PME directement intéressées par le financement public. De façon concrète, la population étudiée regroupe 104 PME devenues sociétés ouvertes et cotées à la Bourse de Montréal à l'époque de la présente étude, soit à l'été 1987. Ces entreprises, oeuvrant dans les secteurs de la fabrication, du détail et des services représentent toutes les entreprises REA enregistrées à la Bourse de Montréal entre 1979 et l'été 1987, dont la valeur nette du capital-actions est évaluée entre un million et quinze millions de dollars, au moment de leur enregistrement sur le marché boursier. Mentionnons que ces critères étaient déterminants pour établir notre échantillonnage.

Un questionnaire 4 de dix-huit pages a été expédié aux PDG de ces 104 entreprises publiques. Par la suite, ils ont reçu deux lettres de rappel, plusieurs appels téléphoniques et nous avons même rencontré personnellement une vingtaine de ces entrepreneurs. Au terme de ces démarches, l'échantillon final se compose de 69 questionnaires remplis, représentant un taux de réponse de $66,3 \%$. Pour chaque cas, le PDG devait désigner, parmi des éléments des six groupes de variables, les changements perçus comme étant les plus importants et préciser si ceux-ci étaient positifs ou négatifs.

Notons enfin qu'un postulat fondamental sous-tend cette typologie : le changement du statut de l'entreprise de fermée à ouverte est mesurable et concluante à trois paliers :

1. celui du comportement du propriétaire d'une PME devenue ouverte;

2. celui de sa gestion et

3. celui de l'entreprise elle-même.

3. Pour obtenir les résultats de l'ensemble de la recherche dont cet article ne constitue qu'une synthèse, voir : Desroches, Jocelyn; Jog, Vijay et al., Les petites et moyennes entreprises et leur financement public au Québec et au Canada : attrait, décision et impact. Cet ouvrage sera publié par l'Institut de recherches politiques du Canada au début de 1990.

4. Le questionnaire divise en quatre parties inclut des questions sur les thèmes suivants :

1. les facteurs influençant la décision d'opter pour la transformation de la société fermée en société ouverte dans le but de financer l'entreprise;

2. les caractéristiques de l'entrepreneur et de l'entreprise;

3. l'impact du financement public;

4. l'influence de différents programmes gouvernementaux sur la décision de devenir une société publique. 


\section{Résultats de cette recherche}

\subsection{Perception globale des effets}

Le tableau 2 montre que les changements perçus par 69 des 104 PDG de PME devenues ouvertes depuis 1984 au Québec l'ont d'abord été sur le plan du statut del'entreprise, puis, par ordre décroissant, sur les plans de la structure de l'entreprise, des communications en général, du processus de décision, du PDG lui-même en tant qu'individu et des relations de travail à l'intérieur de l'entreprise.

Tab. 2. Perception globale des effets du financement publlc sur la PME-Québec (en\%) $(N=69)$

\begin{tabular}{|c|c|c|c|c|c|c|c|}
\hline \multirow[t]{2}{*}{ Eléments } & \multicolumn{7}{|c|}{ Rang* } \\
\hline & 1 & 2 & 3 & 4 & 5 & 6 & Total \\
\hline $\begin{array}{l}\text { PÓLLE EXTERNE } \\
\text { 1. Statut de l'entreprise elle-même }\end{array}$ & 49,2 & 16,9 & 15,4 & 7,7 & 6,2 & 4,6 & 100 \\
\hline PÓLE INTERNE & & & & & & & \\
\hline 2. Restructuration de l'entreprise & 24,2 & 25,8 & 14,5 & 12,9 & 11,3 & 11,3 & 100 \\
\hline 3. Communications en général & 16,7 & 27,3 & 22,7 & 15,2 & 10,6 & 7,6 & \\
\hline 4. Processus de décision & 4,5 & 15,2 & 30,3 & 22,7 & 22,7 & 4,5 & 100 \\
\hline 5. Relations de travail & 1,6 & 3,2 & 4,8 & 15,9 & 27,0 & 47,6 & 100 \\
\hline $\begin{array}{l}\text { PÓLE PERSONNEL } \\
\text { 6. PDG lui-même en tant qu'individu }\end{array}$ & 3,2 & 9,5 & 9,5 & 22,2 & 17,5 & 38,1 & 100 \\
\hline
\end{tabular}

(") Rang $1=10$ premier gros changement...Rang $6=10$ plus petit changement

Les données confirment donc une polarisation certaine sur le plan des effets ressentis par les PDG une fois leur entreprise devenue ouverte. L'effet principal, ressenti par près de $50 \%$ des répondants (soit $25 \%$ supérieur au deuxième choix), a trait au «statut que semble conférer à l'entreprise le monde extérieur une fois celleci devenue publique».

La reconnaissance d'un statut nouveau, conféré par le monde extérieur à la compagnie devenue ouverte, recueille $50 \%$ des choix de premier rang de nos PDG. Deux raisons expliquent le pourcentage très important pour ce pôle externe. Premièrement le fait de devenir ouverte constitue pour une compagnie une évaluation reconnue pour la très grande majorité des cas du professionnalisme, de la qualité de la gestion et des projets actuels et futurs. Deuxièmement, les qualités et intentions de la compagnie devenue ouverte sont largement diffusées à tous les investisseurs potentiels, aux intervenants du monde des affaires et au grand public en général. Cela démontre ainsi une perception de changement dans le statut de l'entreprise. 
Au chapitre de l'influence sur le pôle interne, $47 \%$ des PDG ont relevé un effet considérable en termes de «changements auniveau de la gestion de l'entreprise». À l'intérieur de ce pôle, la variable de changement la plus importante se situe au plan de la restructuration de l'entreprise, alors que les relations de travail sont considérées comme moins importantes.

Il n'est pas surprenant de constater que l'effet ressenti par les PDG sur l'entreprise devenue société ouverte se situe au pôle interne, c'est-à-dire le pôle relié à la gestion interne de l'entreprise. Comme les résultats de notre étude nous le suggèrent, les raisons les plus importantes pour recourir au financement public, sont par ordre décroissant, la possibilité de faire des acquisitions (53\%), d'accroître son fonds de roulement $(37,7 \%)$, de réaliser son projet d'entreprise $(36,2 \%)$ et d'investir dans l'achat ou l'amélioration des équipements et des immobilisations $(30,4 \%)$. Par conséquent, la réalisation d'un ou de plusieurs de ces objectifs apportera des changements considérables dans la gestion interne de la firme, d'où l'explication de $47 \%$ des choix de premier rang pour ce pôle. Toutefois, les entrepreneurs ne perçoivent pas de changement majeur dans les relations de travail même si les employés sont dès lors plus renseignés sur l'entreprise et peuvent de plus participer à sa rentabilité par l'acquisition d'actions appartenant à cette entreprise.

Que le troisième pôle ne retienne que 3,2 \% des choix de premier rang quant à l'effet ressenti à la suite d'un financement public s'explique par le fait que les bénéfices personnels ne sont pas, du moins de façon avouée, un des buts prioritaires du financement public. Il est clair que les changements au sujet du statut de l'entreprise ont une influence indirecte sur l'entrepreneur lui-même, mais il n'y a rien qui ressorte de façon assez évidente pour démontrer que l'entrepreneur considère cette influence comme significative. Ce résultat est surprenantétant donné l'importance de la recherche sur les caractéristiques individuelles ayant trait à ce domaine.

\section{$5 \quad$ Effets sur les composantes de chaque pôle}

Dans cette section, nous décrirons les résultats reliés aux changements qui ressortent de toutes les variables de chacun des trois pôles. Nous avons procédé de façon similaire à la présentation des résultats du tableau 2 en disposant les variables par ordre décroissant d'importance à l'intérieur de chaque pôle. Le tableau 3 fait état de ces résultats.

\subsection{Changement dans le statut de l'entreprise elle-méme}

Majoritairement, comme le montre la section 1 du tableau 3, les PDG s'entendent sur le fait que l'amélioration de l'image de l'entreprise auprès des foumisseurs, des clients, de la communauté d'affaires et du public en général 
Tab. 3. Evaluation des effets du financement public sur la PME - Qub́bec, N = 69

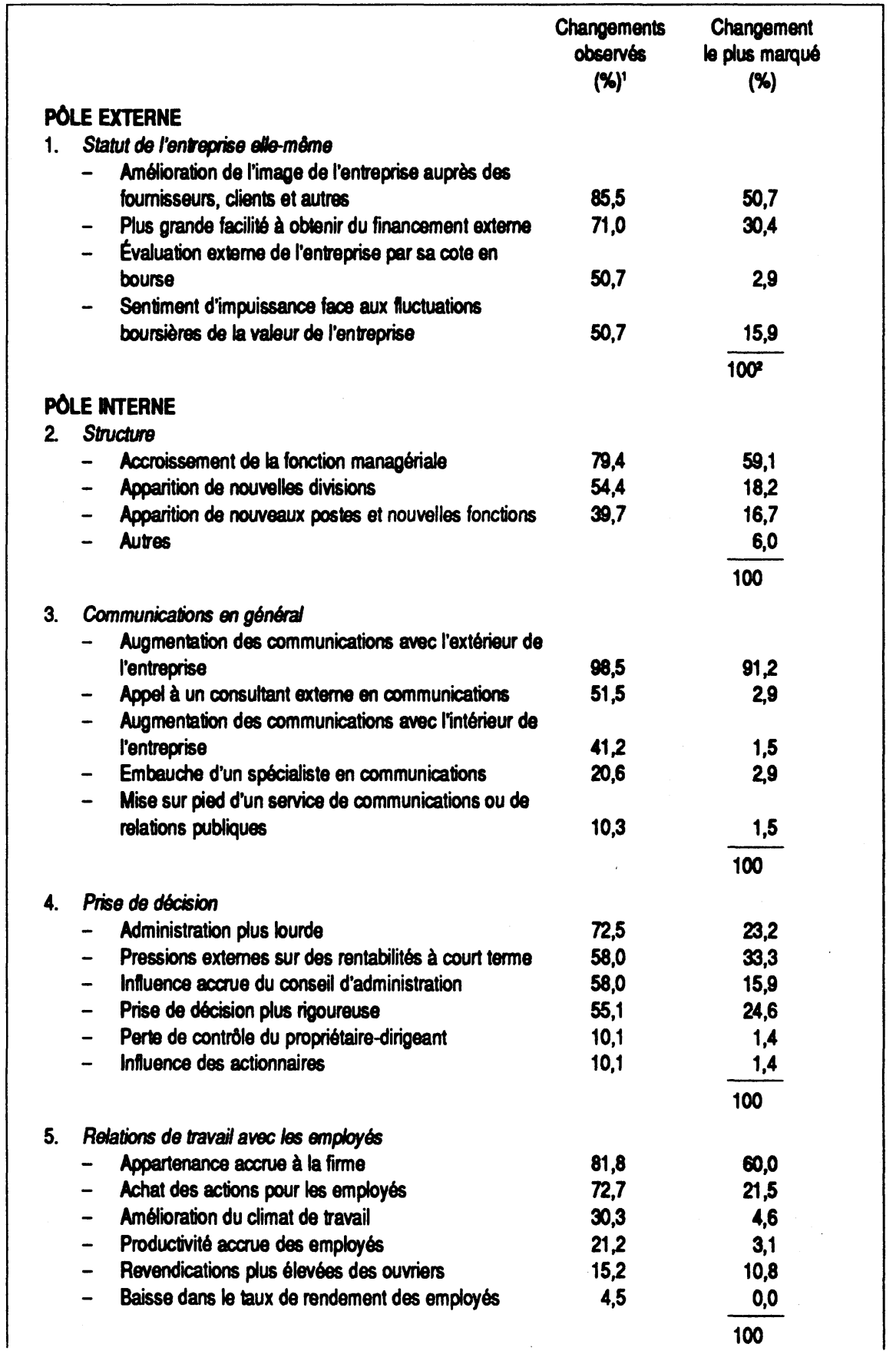


POLE PERSONNEL

6. Dirigeant en tant qu'individu

- Fienté personnelle accrue

$61,5 \quad 20,0$

- Reconnaissance professionnelle accrue

$56,9 \quad 18,5$

- Sentiment d'impuissance face à l'óvaluation marchande de l'entreprise

40,0

33,8

33,8

27,7

18,5

12,3

12,3

$$
\begin{array}{r}
18,5 \\
9,2 \\
12,3 \\
9,2 \\
1,5 \\
7,7 \\
3,1 \\
\hline 100
\end{array}
$$

1 Pour cheque type de variables, les répondants pouvaient choisir un ou plusieurs éléments.

2 Le résultat peut ne pas égaler 100\%, car cortains nombres ont été arrondis.

constitue non seulement l'élément observé le plus fréquemment (dans $85,5 \%$ des cas) mais aussi l'élément où le changement est le plus marqué, c'est-à-dire par $50 \%$ des répondants. À notre avis, la visibilité et la crédibilité acquises par le nouveau statut est l'un des facteurs importants, sinon le plus important. Ainsi, en facilitant l'obtention de sommes d'argent considérables par le biais du financement public, ce statut permet aussi à l'entreprise d'avoir recours à d'autres sources de financement externe sous forme de fonds propres ou de dettes. De plus, selon d'autres résultats de notre enquête, cette accessibilité au financement externe pour couvrir la dette s'avérait difficile ou très difficile pour plus de $41 \%$ de nos entreprises ou encore difficile ou très difficile pour plus de $55 \%$ en ce qui concerne le financement du capital propre; ce changement dans le poble externe est très favorablement accueilli. Non seulement les entreprises reçoivent-elles un financement externe pour couvrir leur avoir, mais elles accèdent plus facilement aux différentes sources de financement pour leur capital futur, élément primordial pour leur croissance et expansion.

En plus de ce bénéfice évident sur le plan financier, $50 \%$ des entrepreneurs ont noté que le financement public s'était avéré, pour eux, un moyen d'évaluation objectif de leur compagnie et, comme pour les deux autres éléments soulignés précédemment, ces changements s'avéraient positifsà $100 \%$. Toutefois, $50 \%$ de nos entrepreneurs ont aussi estimé comme appréciable le sentiment d'impuissance qu'ils ressentent face aux fluctuations boursières des cotes de leur entreprise sur lesquelles ils n'ont que très peu d'influence. Par contre, cetélément n'a été retenu que par moins de $3 \%$ de l'ensemble des entrepreneurs comme étant l'élément le plus important.

\subsection{Changements dans la gestion Interne de l'entreprise}

Afin de mieux cerner les effets d'une société ouverte sur la gestion interne de l'entreprise, quatre domaines de la gestion ont été ciblés spécifiquement. Les questions présentées dans chacun de ces domaines se sont également inspirées de la 
documentation existante en gestion traditionnelle et de certaines suggestions émises dans la phase du test préliminaire. Le tableau 3 (p. 29 et 30), démontre les questions spécifiques posées dans chacun de ces domaines reliés à la gestion de l'entreprise.

Sur le plan structurel, la mise en valeur de la fonction managériale apparait non seulement comme l'élément le plus observé, mais aussi où le changement a été le plus marqué (voir tableau 3, section 2). En outre, presque la moitié des répondants ont déclaré que la transformation de leur entreprise en société ouverte a permis la creation de nouveaux emplois et de nouvelles responsabilités, même si ce fait ne constituait pas un changement important. Certains pourraient émettre l'hypothèse que, dans un premier temps, les effets permettraient la rationalisation de la fonction managériale. Par la suite, ils entraîneraient la création de nouveaux emplois et de nouvelles responsabilités. Avec le temps, ils favoriseraient la création de nouvelles divisions départementales.

Ces changements structurels s'accompagnent inévitablement, comme le montre la section 3 du tableau 3, d'un développement des communications. Que l'intensification des communications avec l'extérieur apparaisse le changement noté et considéré comme le plus marquant par plus de $90 \%$ des PDG va de soi lorsque le statut d'entreprises ouvertes est plus ou moins nouvellement acquis. Néanmoins, la poussée des communications à l'intérieur même de l'entreprise - relevée par plus de $49 \%$ des entrepreneurs - de même que, d'une façon implicite, par une proportion de ceux qui ont observé des modifications dans la structure des communications comme telles - soit par l'embauche d'un spécialiste ou de la mise sur pied d'un service des communications - signifie que les changements internes dans les communications importent mais aussi qu'ils sont moins marquants, moins soudains, plus évolutifs. Cependant, le fait de devenir ouverte implique des modifications significatives dans les communications de l'entreprise aux yeux du monde extérieur.

Nous tournons maintenant notre attention sur les changements apportés au processus de la prise de décision al l' intérieur de l' entreprise publique. La section 4 du tableau 3 montre que pas moins de quatre éléments distincts, chacun se rapportant à une dimension propre de la décision, ont été désignés par $55 \%$ et plus des PDG comme changements importants faisant suite au nouveau statut de leur entreprise. Deux de ces facteurs ont trait à la gestion comme telle qui est perçue par 72,5\% des PDG comme beaucoup plus accaparante, bien que $55,1 \%$ de ces mêmes gestionnaires croient qu'il en découle une prise de décision plus rigoureuse. Il est intéressant de noter que chacun de ces deux facteurs sontégalement considérés comme des changements très prononcés par un nombre d'entrepreneurs à peu près égal, soit 23,2\% et $24,6 \%$ respectivement. Les deux autres facteurs principaux de ce groupe se rapportent aux influences et aux jeux de pouvoir qui découlent du phénomène public. A cet effet, il est intéressant de saisir, comme le montre le même tableau, que les pressions externes sur des rentabilités à court terme sont non seulement observées par $58 \%$ des PDG, mais que ce facteur est en fait considéré comme le changement le plus notable par le plus grand nombre, soit 33,3\%.L'influence duconseil d'administration, 
aussi observée par un nombre identique de $58 \%$ des PDG, diminue toutefois d'intensité à $15 \%$.

Ce qui étonne peut-être autant, comme on le soulignait plus tôt, c'est que la perte de contrôle du propriétaire-dirigeant n'aitété soulignéeque par 10,1\% des PDG et que seulement 1,4\% des PDG l'aient signalée comme changement le plus marqué. Ce résultat, à notre avis, tend à contredire l'énoncé souvent répandu selon lequel le financement public diminue très fortement le contrôle que possède le dirigeant d'entreprise. De plus, les données montrent clairement qu'à la suite de ce changement de statut, il faut tenir compte d'un plus grand nombre d'individus, d'où une gestion plus complexe, mais que la perte de contrôle de l'entreprise ne semble pas une résultante de facto.

Dans les relations de travail avec les employés, l'élément central qui retient l'attention de $60 \%$ des PDG de firmes nouvellement ouvertes est le fait que les employés, pour plusieurs grâce à l'achat d'actions de la compagnie et pour d'autres grâce à une meilleure informations sur leur entreprise, semblent éprouver un sentiment d'appartenance plus grand à l'égard de leur entreprise. Ce sentiment d'appartenance accru recèle certes des conséquences sur le climat de travail, la productivité des employés et les revendications générales des employés, comme l'indique la section 5 du tableau 3.

Par-dessus tout, ces résultats démontrent que devenir une société ouverte implique des changements significatifs dans la façon de gérer l'entreprise. Le changement le plus important provient du palier des communications externes et domine les considérations internes. Il en résulte un développement plus poussé des fonctions managériales et des responsabilités administratives. De plus, les entrepreneurs croient que le fait pour leur entreprise de devenir ouverte a permis le développement du sentiment d'appartenance des employés à l'égard de l'entreprise. Différentes raisons peuvent expliquer cette évolution : par exemple, le nouveau statut de l'entreprise, la possibilité pour les employés d'acquérir des actions de l'entreprise, ou simplement l'accès à une plus grande disponibilité et diffusion de l'information. Malheureusement, à la suite du sondage, cette interrogation demeure sans réponse catégorique.

\subsection{Changements concernant les caractéristiques du PDG}

La section 6 du tableau 2 montre que les trois variables intimement reliées au sentiment de reconnaissance et d'accomplissement ressenti par le PDG en regard de son entreprise ouverte-soit la fierté personnelle, la reconnaissance professionnelle et le statut social - ressortent le plus souvent et sont considérées comme les plus marquantes. Le prix de cette reconnaissance personnelle n'est toutefois pas à sousestimer puisqu'il se fait aux dépens d'une perte de disponibilité envers la famille, envers les employés et de la perte de certains avantages personnels en ce qui concerne les comptes de dépenses personnelles, cela conformément à la théorie des coûts 
d'agence. Cependant, ces divers facteurs ne comptent pas vraiment parmi ce qui marque le plus le PDG d'une entreprise nouvellement ouverte. L'élément qui semble l'affecter le plus et pour lequel il se sent impuissant, c'est son incapacité à pouvoir influencer personnellement le cours des actions de l'entreprise à la Bourse, qui, selon lui, reflète rarement la valeur réelle des actifs ou de la gestion de l'entreprise.

\section{La direction des changements}

Jusqu'ici, nous avons concentré notre attention sur le degré de changement effectué sur le pôle personnel et la perception qu'en ont eue les entrepreneurs. Mais certaines questions évidentes et intéressantes demeurent sans réponse. Ainsi, ces entrepreneurs perçoivent-ils positivement ou négativement leur décision d'avoir transformé leur entreprise en compagnie ouverte ? Dans quels pôles trouvent-ils leur appui ? Et lequel de ces pôles les rend heureux ou insatisfaits? Le tableau 4 montre le taux de «positivité» ou de perception positive associée à chacun des six groupes de variables.

Tab. 4. Perception globale des changements du financement public sur la PME ot le degré de perception positive associée da ces changements - (Québec, $N=69$ )

\begin{tabular}{|lcc|}
\hline PÓLES & $\begin{array}{c}\text { Importance } \\
\text { du changement } \\
\text { Groupes de variables }\end{array}$ & $\begin{array}{c}\text { Perception positive } \\
\text { du changement } \\
(\%)\end{array}$ \\
\hline POLE EXTERNE & & \\
1. Statut de l'entreprise elle-même & 49,2 & 83,6 \\
POLE INTERNE & & \\
2. Structure & 24,2 & 94,1 \\
3. Communications en général & 16,7 & 89,2 \\
4. Prise de décision & 4,5 & 58,5 \\
5. Relations de travail avec les employés & 1,6 & 89,1 \\
POLE PERSONNEL & & \\
6. Dirigeant en tant qu'individu & 3,2 & 65,6 \\
\hline
\end{tabular}

Les résultats du tableau 4 ne laissent pas d'étonner. En effet, ils démontrent que les changements les plus marqués pour chacun des six groupes de variables sont très positifs. Ce tableau indique que le recours au financement public influence positivement tant l'entreprise, la gestion interne que son PDG lui-même. 
Ainsi, le statut de l'entreprise qui connaît le plus fort taux de changement est perçu globalement comme un changement positif à $84 \%$. Seul le sentiment d'impuissance face aux fluctuations boursières a ramené le taux au-dessous du taux maximal de $100 \%$.

Étant donné qu'il s'agit des effets sur la gestion interne de l'entreprise, les changements les plus prononcés, qui ont été de type structural et qui ont touché les communications en général, présentent aussi des taux de positivité très élevés, soit $94 \%$ et $89 \%$ respectivement. Les relations de travail, qui semblent le moins affectées par le changement de statut de l'entreprise, n'en montrent pas moins un taux de positivité de $89 \%$; seules les revendications plus importantes des employés rabaissent ce taux en bas du taux maximal de $100 \%$. Pour sa part, le processus de décision semble être la dimension la plus problématique puisque, bien que positive à près de $60 \%$, la lourdeur administrative semble être extrêmement difficile à porter.

Quant au PDG lui-même, la fierté personnelle et professionnelle qu'il en retire de même que son statut social sont satisfaisantsà $100 \%$. Toutefois, le prix qu'il doit payer sur le plan familial et autres font descendre le taux global à $65 \%$.

\section{Conclusion}

L'intention principale de cette étude est de fournir un résultat manifeste des effets du financement public sur les trois pôles reliésà l'entreprise età l'entrepreneur. Cette recherche fut effectuée par le biais d'un questionnaire expédié aux PDG de petites et moyennes entreprises devenues ouvertes grâce au RÉA.

Les résultats de cette recherche sont très logiques. Ils démontrent clairement que la transformation d'une société fermée en société ouverte apporte des changements significatifs au chapitre du statut de l'entreprise, de sa gestion interne et du PDG luimême. Toutefois, ces résultats mettent en doute certaines croyances populaires concernant ces changements. En premier lieu, les changements les plus significatifs se reflètent surtout au sujet du statut de l'entreprise. Ainsi, le statut public de l'entreprise améliore la perception des gens à l'extérieur, facilite la gestion et permet une plus grande accessibilité pour l'entreprise aux marchés financiers. Les communications externes et internes sont aussi affectées de façon notable. Ainsi, l'entrepreneur qui décide d'orienter son entreprise vers le marché du financement public doit prendre en considération le besoin plus considérable d'information et de communication. Parmi ces différents changements se trouvent aussi de nouvelles charges administratives et des fonctions managériales accrues. Les effets sur les employés sont loin d'être négligeables; leur sentiment d'appartenance semble plus aiguisé. C'est, au demeurant, la conclusion partagée par la majorité des PDG. Le pôle personnel, c'est-à-dire les effets sur le PDG, est, des différents pôles, celui qui accuse 
le moins d'influence. Toutefois, les PDG consultés déclarent avoir ressenti une plus grande fierté personnelle ainsi qu'une reconnaissance professionnelle plus élevée.

Deux tendances principales se dégagent des résultats de cette enquête. Premièrement, la décision de transformer une société fermée en société ouverte implique non seulement un flux monétaire immédiat en capital net, mais aussi un changement positif et permanent de l'environnement externe et financier de l'entreprise.Lefinancement public peut constituer lapremière manceuvre d'importance vers la croissance et la maturité des PME. Deuxièmement, cette expérience est très positive. S'il y a certains désavantages, cette recherche ne nous a pas permis de les mettre à jour. Par conséquent, cela suppose certainement que, si les gouvemements sont sérieux au sujet de l'amélioration de l'environnement des PME, ils doivent poursuivre leur encouragement auprès des entrepreneurs afin que ceux-ci s'engagent plus facilement dans une telle orientation. Les differents aspects que pourrait prendre cet encouragement dépendront de la philosophie du gouvernement et de sa politique fiscale.

\section{BIBLIOGRAPHIE}

Arnold, J. L., (1985), «Exempt offerings : going public privately», Harvard Business Review, vol. 63, pp. 16-29.

Bamberger, J., (1986), «Values and strategic ehaviour , Management International Review, vol. $26, n^{\circ} 4$, pp. 57-69.

Brigham, E. et Smith, K., (1975), «The cost of capital to the small firm». Dans Emest Waller, (ed.). The Dynamic of Small Firm, Chapitre 10. Austin, Texas, Austin Press.

Bulloch, J., (1985), Renforcer l' esprit d'entreprise au Canada. Discours prononcé à la 30 conférence annuelle mondiale du Conseil international de la petite entreprise, Montréal, 18 juin, 18 p.

Chiasson, C., (1986), «Demière semaine pour acheter des actions dans le cadre du REA en 1986», Les Affaires, 20 décembre, pp. 42-43.

Coallier, R., (1988), A Study of the Attitudes of Quebec Entrepreneurs toward Initial Public Offerings. A business research project submitted in partial fulfillment for the degree of M.B.A. at Concordia University. Montreal, mai, 60 p.

Commission québécoise sur la capitalisation des entreprises, (1984), Rapport au ministre de l'Industrie, du commerce et du tourisme, Gouvernement du Québec. 2 trimestre 1984, $99 \mathrm{p}$.

Conseil économique du Canada, (1982), «Business assistance», Intervention and Efficiency. A Study of Government Credit and Credit to the Private Sector. Ottawa, Ministry of Supply and Services, chapitre 3, pp. 17-41.

De Vries, M., (1977), «The entrepreneurial personality : A person at the crossroads»,Journal of Management Studies, vol. 14; pp. 34-57.

Dubé, D., (1988), «Les REA abandonnés» Cahier spécial du journal Finance, $1^{\text {er }}$ øût, pp. 17 24.

Dun \& Bradstreet Canada Limited, (1986), An Analysis of The Need for an Acquisition of Business Funds Among Medium Sized Corporations in Canada. Toronto, Marketing Services Division, $21 \mathrm{p}$. 
Gasse, Y., (1976), Entrepreneurs' Cybernetic Predispositions, Business Ideology and Effectiveness of Small Organizations in TwoIndustrial Environments, Ottawa, Department on Industry, Trade and Commerce.

Gasse, Y., (1979), Attitudes of Canadian Entrepreneurs Towards Outsiders Investing inTheir Business Ventures. Document spécial, Université Laval, Faculté des Sciences de l'administration.

Gasse, Y., (1982), «Elaborations on the psychology of the entrepreneur», dans D.L. Sexton, (ed.). Encyclopedia of Entrepreneurship. Englewood Cliffs, New Jersey, Prentice Hall.

Gasse, Y.,(1984a), «Attitudes et prédispositions des propriétaires-dirigeants et entrepreneurs canadiens envers les bailleurs de fonds externess. Document de travail, Université Laval; Faculté des Sciences de l'administration.

Gasse, Y., (1984b), «Attitudes toward external financing : A comparison of canadian entrepreneurs and owner-managers», dans John A. Hornaday (ed.). Frontiers of Entrepreneurship Research, Boston, Center for Entrepreneurial Studies, Babson College.

Gasse, Y., (1985), «L'entrepreneurship : une stratégie de recherche et d'intervention pour le développement», P.M.O., vol. 1, $\mathrm{n}^{\circ}$ 5, pp. 8-24.

Guertin, J., (1984), «Les PME sont-elles sous capitalisées ?», dans La commission québécoise sur la capitalisation de l' entreprise, pp. 56-65.

Henri, J., (1987), «Les petites entreprises RÉA se font plus nombreuses qu'en 1986», Les Affaires, 8 août, p. 26.

Kostuch, M., (1976), «Cashing in on the new entrepreneurial climate. Part II - Sources of help and financing», Business Quarterly, vol. 41, pp. 61-73.

Liao, S. S., (1974), «The effect of the size of firms on managerial attitudes», California Management Review, vol. 18, pp. 59-65.

Martin, M., (1987), Shareownership in Quebec, 1987. Montréal, Commission des valeurs mobilières du Québec, Service de recherches, juin.

O'Neill, H. M., (1983), «How entrepreneurs manage growth», Long Range Planning, vol. 16; $\mathrm{n}^{\circ} 1, \mathrm{pp} .116-123$.

Riverin, B., (1988a), «Le krash boursier du 19 octobre 1987 un an après...», Montréal, Bourse de Montréal, 13 octobre, $25 \mathrm{p}$.

Riverin, B., (1988b), «Le REA, un investissement boursier très rentable, un facteur de développement économique puissant». Allocation présentée à la Chambre de Commerce de Montréal, le 23 février, $26 \mathrm{p}$.

Robert, P., (1983), «Government intervention in business financing», Economic Council of Canada, Ontario, Niagara-on-the-lake, The Niagara Institute, $30 \mathrm{p}$.

Sécor Inc., (1986), L'évaluation du Régime d' épargne-actions du Québec. Montréal, novembre, $87 \mathrm{p}$.

Sécor Inc., (1988), Les titres RÉA et le marché secondaire. Montréal, mars, 38 p.

Smith, D., (1984), Pourquoi la petite entreprise est si importante. Brochure publiée par le ministère d'État aux petites entreprises et tourisme, Ottawa, 8 p.

Steinmetz, L. L., (1969), «Critical stages of small business growth when they occur and now to survive them», dans The Entrepreneur's Handbook II, pp. 33-40.

Tardif, M., (1985), «On small and medium-sized businessesw. A speech to the 30th world conference on small business, Montréal, 17 juin, 11 p.

The Financial Times of Canada, (1985), «QSSP a big hit for firms», 15 avril.

The Globe and Mail, (1984), «Quebecers flock to tax shelters», 13 octobre.

The Toronto stock exchange, (1980), «Nothing ventured... Iivesting in Canada's winners». Toronto, $65 \mathrm{p}$. 
Thornton, D. R., (1981), «Small Business financing policies and practices : from interview survey of manager», dans Small Business Financing and Non-Bank Financial Institution, vol. I. pp. 32-125.

Toulouse, J., (1979), L'entrepreneurship au Québec, Montréal, Fides.

Toulouse, J., (1984), «L'entrepreneurship, son entreprise et le financement de celle-ci». Texte présenté à la Commission sur la capitalisation. Montréal, avril, $27 \mathrm{p}$.

Wynant, L. et al., (1982), Chartered Bank Financing of Small Business in Canada. London, Ontario, University of Westem Ontario, School of Business Administration. 